Revista de Matemática: Teoría y Aplicaciones 1998 5(2) : 87-112

CIMPA - UCR - CCSS ISSN: 1409-2433

\title{
ISOMORFISMO DE GRAFO Y DE FUNCIONES LÓGICAS CON ALGUNAS APLICACIONES
}

\author{
(Mijail Bulat)
}

3 de junio de 2004

Resumen

Se propone un método de solución del problema de isomorfismo para grafos que permite reducir escencialmente el sondeo de variantes durante el proceso de solución. El métode se aplica para cualesquiera grafos (dirigidos, no -dirigidos, pesados y etc.) y hipergrafos.con algunas midificaciones el método se aplica para resover el mismo problema para funciones lógicas.

Se examinan unas aplicaciones:

a) la busqueda dde los ciclos (cadenas) hamilotonianos,

b) la solución del problema de Frobenius para matrices fuertemente equivalentes,

c) la codificación de los estados interiores de la máquina finita.

Abstract

Method to solve the isomorphism problem for graphs is suggested, which significantly decreases the number variants to be checked. The method is applicable to any graphs (directed, undirected, meighted etc.) and hipergraphs. With some modifications it can be applied for solving isomorphism problem for logical functions.

Some applications are considered:

a) search for hamiltonian cycles (paths),

b) solutionFrobenius problem for strongly equivalent matrices,

c) coding inside states of finite automate.

1. Introducción

El número de grafos distintos que podemos construir con solo alglunos vértices y aristas es enorme y de hecho este número se transforma en astronómico rapidamente cuando incrementamos el número de vértices y de aristas permitidos. Sin embargo unos grafos difieren entre si solamente porque sus vértices son etiquetados de manera diferente. Reetiquetando los vértices de uno de estos grafos se obtiene el otro. En estos casos se dice que los grafos son isomorfos. En [5], [6], [7], [8] se indica que el problema de isomorfismo de grafos es díficil ya está vinculado con un gran sondeo de variantes. Los métodos de 
investigación que existen están basándose en las particularidades específicas de los grafos y por eso sirven para unos grafos y no para todos.El mismo problema hay en las funciones lógicas que están en la base de los circuitos lógicos.

Una cantidad se llama invariante bajo isomorfismo si su valor es el mismo para cualesquiera dos grafos isomorfos. Invariantes son: el número de vértices o de aristas, la suma de los elementos de cada una de las filas (columnas) de la matriz de adyacencias, el determinante de ésta matriz, la densidad deñ grafo, los ciclos o cadenas de una longitud determinada y etc. Pero con frecuencia sucede que el cálculo de los invariantes es mas complicado que el problerma de isoformismo. En esta situación estamos, por ejemplo, cuando usamos como invariante la densidad de4l grafo los ciclos hamiltonianos. Estos invariantesm tienen gran importancia en la solución de diversos problemas. Como se muestra en [8] sería mejor reducir el problema delo cálculo de los invariantes importantes al problema de isomorfismo y no al revés. De lo expuesto resulta que se necesita un método eficaz de la solución del problema de isomorfismo con invariantes que se calculen fácil y que sreviría para todos los tipos de grafos.

2. Grafos isomorfos

Dados dos grafos $G=(X, E)$ y $G^{*}=\left(X^{*}, E^{*}\right)$ y $f: x \rightarrow x^{*}$ es una aplicación biyectiva entre los conjuntos de vértices tal que $\left\{x_{i}, x_{j}\right\}$ es una arista de $G$ si y solo si $\left\{f\left(x_{i}\right), f\left(x_{j}\right)\right\}$ es una arista de $G^{*}$. Entonces $f$ se llama isomorfismo entre $G$ y $G$,y se dice que $G$ y $G^{*}$ son isomorfos y se designa $G \cong G^{*}$.Es evidente que si $|X| \neq\left|X^{*}\right|_{\mathrm{o}}|Y|=\left|Y^{*}\right|$. Y además $X=X^{*}=\left\{x_{l}, \ldots, x_{n}\right\}$ e $Y=Y^{*}=\left\{y_{l}, \ldots, y_{m}\right\}$. Sea $A_{G}=\left(a_{i j}\right)_{n \times n}$ y $A_{G^{*}}=\left(a_{i j}^{*}\right)_{n \times n}$ las matrices de adyacecias de los grafos $G$ y $G^{*}$ respectivamente.

$$
A_{G}=
$$

Se sabe [5], [6], [7] , [8] que $G \cong G^{*}$ si y solo si existe alguna sustitución $S=\left(\begin{array}{c}x_{l} \ldots x_{n} \\ x_{i_{1}} \ldots x_{i_{n}}\end{array}\right), i_{l}, \ldots i_{n} \in$ $\{l, \ldots n\}$ por medio de la cual una de las matrices, por ejemplo, $A_{G}$, se tranforma a la otra. En este caso designaremos

$$
A_{G}(S)=A_{G^{*}}(1)
$$

En la sustitucíon $S$ la primera fila correponde a los vértices de $G$ y la segunda a los de $G^{*}$. El problema de investigación de dos grafos sobre isomorfismoconsiste en la búsqueda de la sustitución $S$ según la cual se cumpla (1). Si tal sustitución no existe entones los grafos no son isomorfos.

Describiremos un método de investigación sobre isomorfismo que permite resolver el problema para cualquier par de grafos independientemente de las particularidades específicas de los mismos usando siempre el mismo procedimiento. Veamos la matriz $A_{G}$ cuyos elementos son números enteros. Compongamos grupos $R_{G, i}(i=l, \ldots, p)$ de filas con misma suma de elementos de cada una. Escribimos los grupos compuestos uno bajo otro en el orden creciente de las sumas indicadas, separándolos por líneas horizontales $\left(T_{1}, T_{2}, T_{3}\right)$. Componemos la matriz $B_{G}=\left(b_{i j}\right)_{p \times n}$

$B_{G}=$

donde $p$ es el número de grupos compuestos, $b_{i j}$ es igual a la suma de los elementos de la j- ésima columna en el i- ésimo grupo $R_{G ; i}$

Designemos por $C_{j, G}$ la j- ésima columna de $B_{G}$ y por $\lambda_{j, G}$ su multiplicidad. Análoga- 
mente por la matriz $A_{G^{*}}$ construimos los grupos $R_{G^{*}, i}(i=1, \ldots, r)$ y dspués la matriz $B_{G^{*}}$ y harmos las mismas designaciones $C_{j, G^{*}}$ y $\lambda_{j, G^{*}}$. Ya que $p,\left|R_{G, i}\right|, C_{j, G}$ y $\lambda_{j, G}$ son invariantes entonces para que $G \cong G^{*}$ es necesario que se cumplan:

$$
\begin{aligned}
& p=r(2) \\
& \left|R_{G, i}\right|=\left|R_{G^{*}, i}\right|(3) \\
& \forall i \in\{1, \ldots, n\} \exists j \in\{1, \ldots, n\} \text { tal que } C_{i, G}=C_{j, G^{*}} \text { y } \lambda_{i, G}=\lambda_{j, G^{*}}
\end{aligned}
$$

Veamos dos casos particulares:

a) $\lambda_{i, G}=\lambda_{j, G^{*}}=1$ En este caso las columnas $C_{i, G}$ y $C_{j, G}$ en las matrices $B_{G}$ y $B_{G^{*}}$ rspectivamente son simples y en la sustitución $S$ al vértice $x_{i}$ de $G$ corresponde el vértice $x_{j}$ de $G^{*}$ y escribiremos $x_{i} \leftrightarrow x_{j}$.

b) $\lambda_{i, G}=\lambda_{j, G^{*}} \neq 1$ En tal caso $C_{i, G}$ y $C_{j, G}$ son múltiples y al vértice $x_{i}$ puede corresponder cualquier vértice $x_{j}$ para los cuales se cumplen (4). Por consiguiente, en la sustitución $S$ al subconjunto de vértices de $G$ se pone en correspondencia un subconjunto de vértices de $G^{*}$.

En el caso general de las matrices $B_{G}$ y $B_{G^{*}}$ corresponden la sustitución

$$
\widetilde{S}=\left(\begin{array}{c}
x_{a_{1}} \ldots x_{a_{t}}\left\{x_{b_{1}}, \ldots, x_{b_{k}}\right\} \ldots\left\{x_{c_{1}}, \ldots, x_{c_{s}}\right\} \\
x_{d_{1}} \ldots x_{d_{t}}\left\{x_{e_{1}}, \ldots, x_{e_{k}}\right\} \ldots\left\{x_{f_{1}}, \ldots, x_{f_{s}}\right\}
\end{array}\right)
$$

donde $x_{a_{1}}, \ldots, x_{a_{t}}, x_{d_{1}}, \ldots, x_{d_{t}}$ corresponden a las columnas simples y los demás - a las columnas múltiples con las multiplicidades $k, \ldots, s\left(a_{1}, \ldots, f_{s} \in\{1, \ldots, n\}\right)$.

De esta manera por msdio de las matrices $B_{G}$ y $B_{G^{*}}$ se obtienen dos particiones sobre el conjunto de vértices y se establece una correspondencia entre los subconjuntos de esetas particiones. Si $x_{i}$ corresponde a una columna de multiplicidad $k$ entonces diremos que $x_{i}$ es vértice de multiplicidad $k$ de la participación respectiva. Si la sustitución contiene vértices múltiples entonces esta se llama susutitución múltiple y se designa por $\widetilde{S}$. Si todos los vértices de la sustitución son simples entonces esta se llama sustitución simple y se designa por $S$.

Hagamos las designaciones siguientes:

$$
M_{1}=\left\{x_{b_{1}}, \ldots, x_{b_{k}}\right\}, \ldots, M_{q}=\left\{x_{c_{1}}, \ldots, x_{c_{k}}\right\}, M_{1}^{*}=\left\{x_{e_{1}}, \ldots, x_{e_{k}}\right\}, \ldots, M_{q}^{*}=\left\{x_{f_{1}}, \ldots, x_{f_{k}}\right\}
$$

$$
\stackrel{\tilde{S}}{\text { Entonces }}=\left(\begin{array}{l}
x_{a_{1}} \ldots x_{a_{t}} M_{1} \ldots M_{q} \\
x_{d_{1}} \ldots x_{d_{t}} M_{1}^{*} \ldots M_{q}^{*}
\end{array}\right)
$$

Las condiciones (2),(4) son solamente necesarias de la existencia del isomorfismo. Si existe la sustitución $S$ se obtiene de $\widetilde{S}$ para la cual se cumpla (1) entonces $G \cong G^{*}$.

Ejemplo 1 Investigar cuales de los grafos $G_{1}, G_{2}, G_{3}$ de la fig.1 son isomorfos entre sí.

Solución Componemos las matrices de adyacencias $A_{G_{1}}, A_{G_{2}}, A_{G_{3}}$; los grupos de las filas (las tablas $\mathrm{T}_{1}, \mathrm{~T}_{2}, \mathrm{~T}_{3}$ ) y las matrices $B_{G_{1}}, B_{G_{2}}, B_{G_{3}}$. Las condiciones (2) y (3) se cumplen para todas las tablas $\mathrm{T}_{1}, \mathrm{~T}_{2}$ y $\mathrm{T}_{3}$ pero la condición (4) se cumple solamente para $B_{G_{1}}$ y $B_{G_{2}}$. De estas matrices resulta la sustitución $\tilde{S}$ de la cual se obtienen dos ssustituciones simples $S_{1}$ y $S_{2}$.

Para $S_{1}$ se cumple la condición (1), por eso $G_{1} \cong G_{2}$.

Los vértices simples de la sustitución $\widetilde{S}$ a su vez pueden engendrar nuevos vértices simples. 
Veamos el caso más general cuando los elememtos de las matrices $A$ son símbolos arbitrarios. En este caso no siempre podemos construir las matrices $B$. Supongamos que las sustituciones (5) y (6) se obtienen según ciertas propiedades de las filas y columnas de las matrices $A$.

Componemos la matriz $C_{M_{1}}=\left(c_{i j}\right)_{t \times k}$, donde $c_{i j}=a_{a i b j}$, y $a_{i}, b_{j} \in\{1, \ldots, n\}$

Análogamenteconstruimos todas las matrices $C_{M i}, C_{M i}^{*}(i=1, \ldots, q)$. Las columnas de las matrices $C$ son invariantes. Si $G \cong G$ entonces para cada par de matrices $\left(C_{M i}, C_{M i}^{*}\right)$ tienen que cumplirse la condición (4). Si la condición (4) no se cumple por lo menos oara un par de matrices entonces los grafos no son isomorfos.

Todas las matrices $C$ pueden incluirse en una tabla gebneral $\mathrm{T}_{4}$.

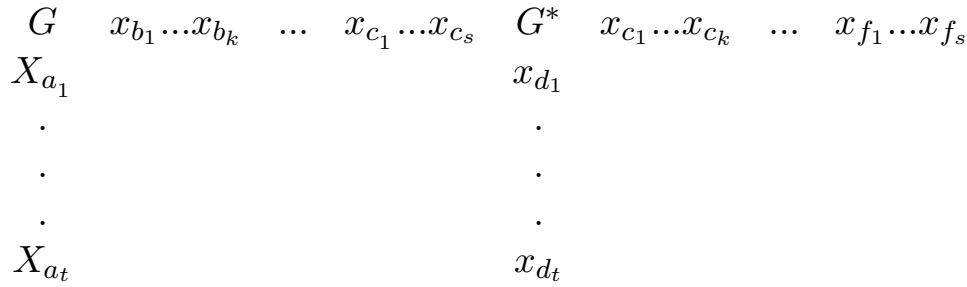

Losd vértices simples se escriben en las columnas $G$ y $G^{*}$. En la misma fila de estas columnas se escriben los vértices que corresponden uno a otro en $\widetilde{S}$. La tabla general de $\widetilde{S}$ del Ejemplo es $\mathrm{T}_{5}$.

$\begin{array}{cccc}G_{1} & x_{4} x_{5} & G_{2} & x_{1} x_{8} \\ x_{1} & 00 & x_{5} & 00 \\ x_{2} & 00 & x_{4} & 00 \\ x_{3} & 10 & x_{3} & 10 \\ x_{6} & 00 & x_{6} & 00 \\ x_{7} & 00 & x_{2} & 00 \\ x_{8} & 00 & x_{7} & 00 \\ x_{4} & & x_{1} & \\ x_{5} & & x_{8} & \\ & & T_{5} & \end{array}$

De $\mathrm{T}_{5}$ resulta que $S_{1}=\left(\begin{array}{l}x_{1} x_{2} x_{3} x_{4} x_{5} x_{6} x_{7} x_{8} \\ x_{5} x_{4} x_{3} x_{6} x_{2} x_{7} x_{1} x_{8}\end{array}\right)$ y esta es la única susutitución para la cual se cumple (1).

Si después de la comparación de los pares de matrices $C$ aparecen nuevos vértices simples, entonces continuamos la construcción de la tabla general $\mathrm{T}_{4}$ en la parte de abajo separándola de la parte ya construida por una línea horizontal o construimos una nueva tabla general con los nuevos vértices simples y nuevos subconjuntos $M$.

Supongamos que las matrices $C$ no engendran nuevos vértices simples. En este caso podemos usar otra matriz. Sea $M_{i}=\left\{x_{i}, \ldots, x_{i_{h}}\right\}$ un subconjunto $\widetilde{S}$ y $X_{j}$ un vértice de esta misma. Definimos una función $\tau\left(M_{i}, X_{j}\right)$ de modo siguiente:

$$
\tau\left(M_{i}, X_{j}\right)=\left|u=\stackrel{h}{U}\left\{a_{i_{u} j}\right\}\right|
$$


Por medio de esta función construimos la matriz $D_{M_{1}}=\left(d_{i j}\right)_{q \times k}$, donde $d_{i j}=\tau\left(M_{i}, x_{b_{j}}\right)$

Análogamente construimos las demás matrices $D_{M_{2}}, \ldots, D_{M_{q}}, D_{M_{1}^{*}}, \ldots, D_{M_{q}^{*}}$. Las columnas de estas matrices son invariantes bajo isomorfismo. Por eso si $G \cong G^{*}$ entonces para culquier par de matrices $\left(D_{M_{1}}, D_{M_{1}^{*}}\right)$ deben cumplirse las condiciones (4). Todas las matrices $D$ pueden incluirse en una tabla general $\mathrm{T}_{6}$.

\begin{tabular}{cccccccc}
$G$ & $x_{b_{1}}, \ldots, x_{b_{k}}$ & $\ldots$ & $x_{c_{1}}, \ldots, x_{c_{s}}$ & $G^{*}$ & $x_{e_{1}}, \ldots, x_{e_{k}}$ & $\ldots$ & $x_{f_{1}}, \ldots, x_{f_{s}}$ \\
$M_{1}$ & $\cdot$ &. & $\cdot$ & $M_{1}^{*}$ & $\cdot$ & $\cdot$ & $\cdot$ \\
$\cdot$ & $\cdot$ & $\cdot$ & $\cdot$ & $\cdot$ & $\cdot$ & $\cdot$ & $\cdot$ \\
$\cdot$ & $\cdot$ & $\cdot$ & $\cdot$ & $\cdot$ & $\cdot$ & $\cdot$ & $\cdot$ \\
$\cdot$ & $\cdot$ & $\cdot$ & $\cdot$ & $\cdot$ & $\cdot$ & $\cdot$ & $\cdot$ \\
$M_{q}$ & $\cdot$ & $\cdot$ & $\cdot$ & $M_{q}^{*}$ & $\cdot$ & $\cdot$ & $\cdot$ \\
$\sum_{\tau}$ & & & \multicolumn{5}{c}{$T_{6}$}
\end{tabular}

En la última fila de esta tabla se escribe la suma de los elementos de cada columna que la usaremos en los sucesivo.

Las matrices $D$ pueden engendrar nuevos vértices simples o transformar los sbconjuntos $M$ y las sustituciones $\widetilde{S}$. Si aparecen nuevos vértices simples entonces construimos las matrices $C$ y después, si es necesario, otras matrices $D$.

Si por medio de las matrices $C$ y $D$ la sustitución $\widetilde{S}$ no cambia entonces esta se llama sustitución estable. En el caso contrario - inestable.

Supongamos que $\widetilde{S}$ es estable. En este caso hay que considerar que culquier vértice $x_{i} \in$ $M_{r}$ corresponde a un vértice $x_{j} \in M_{r}^{*}(r=1, \ldots, q)$, es decir $x_{i}$ e $x_{j}$ son simples. Con esta condición construimos las matrices $C$ y destacamos, si es posible, nuevos vértices simples. Si las condiciones (4) no se cumplen por lo menos para un par de matrices $C$ entonces $x_{i}$ no corresponde a $x_{j}$ y hacemos otra suposición. Si de nuevo se obtiene una sustitución estable entonces usamos otro par de vértices simples y continuamos la construcción de las matrices $C$ y $D$. Para acelerar el proceso de transformación de las sustituciones $\widetilde{S}$ hace falta tomar en calidad de vértice simple aquel vértice $x_{j}$ para el cual $\sum_{\tau}\left(M_{i}, X_{j}\right)$ es máxima. Si para algún par de matrices $D$ no se cumplen las condiciones (4) entonces hay que regrsar a la susutitución anterior $\widetilde{S}$ y hacer otra suposición.

Hace falta señalar que apartir de un momento todas las sustituciones $\widetilde{S}$ son inestables y solamente con las matrices $C$ sin falta se obtiene la susutitución simple $S$.

Ejemplo 2. Investigar sobre isoformismo los grafos $G$ y $G^{*}$ de la Fig. 2.

Solución. los grafos son 3-regulares. Construimos las matrices de adyacencias $A$ y después las matrices $B$.

$\begin{array}{llllllllll}x_{1} & x_{2} & x_{3} & x_{4} & x_{5} & x_{6} & x_{7} & x_{8} & x_{9} & x_{10}\end{array}$ 


$$
\begin{aligned}
& A_{G}=\begin{array}{c}
x_{1} \\
x_{2} \\
x_{3} \\
x_{4} \\
x_{5} \\
x_{6} \\
x_{7} \\
x_{8} \\
x_{9} \\
x_{10}
\end{array}\left[\begin{array}{llllllllll}
0 & 0 & 1 & 1 & 0 & 0 & 1 & 0 & 0 & 0 \\
0 & 0 & 0 & 1 & 1 & 0 & 0 & 1 & 0 & 0 \\
1 & 0 & 0 & 0 & 1 & 0 & 0 & 0 & 1 & 0 \\
1 & 1 & 0 & 0 & 0 & 0 & 0 & 0 & 0 & 1 \\
0 & 1 & 1 & 0 & 0 & 1 & 0 & 0 & 0 & 0 \\
0 & 0 & 0 & 0 & 1 & 0 & 0 & 1 & 1 & 0 \\
1 & 0 & 0 & 0 & 0 & 0 & 0 & 0 & 1 & 1 \\
0 & 1 & 0 & 0 & 0 & 1 & 0 & 0 & 0 & 1 \\
0 & 0 & 1 & 0 & 0 & 1 & 1 & 0 & 0 & 0 \\
0 & 0 & 0 & 1 & 0 & 0 & 1 & 1 & 0 & 0
\end{array}\right] \\
& \begin{array}{llllllllll}
x 1 & x 2 & x 3 & x 4 & x 5 & x 6 & x 7 & x 8 & x 9 & x 10
\end{array} \\
& \begin{array}{lllllllllll}
x 1 & 0 & 1 & 0 & 0 & 0 & 0 & 0 & 1 & 0 & 1
\end{array} \\
& \begin{array}{lllllllllll}
x 2 & 1 & 0 & 1 & 0 & 0 & 0 & 1 & 0 & 0 & 0
\end{array} \\
& \begin{array}{lllllllllll}
x 3 & 0 & 1 & 0 & 1 & 0 & 1 & 0 & 0 & 0 & 0
\end{array}
\end{aligned}
$$

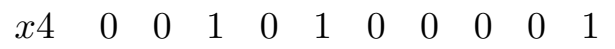

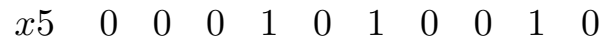

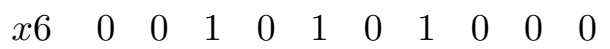

$$
\begin{aligned}
& \begin{array}{lllllllllll}
x 7 & 0 & 1 & 0 & 0 & 0 & 1 & 0 & 0 & 1 & 1
\end{array}
\end{aligned}
$$

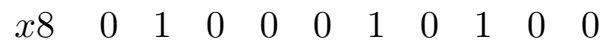

$$
\begin{aligned}
& \begin{array}{lllllllllll}
x 9 & 0 & 0 & 0 & 0 & 1 & 0 & 0 & 1 & 0 & 1
\end{array} \\
& \begin{array}{lllllllllll}
x 10 & 1 & 0 & 0 & 1 & 0 & 0 & 0 & 0 & 1 & 0
\end{array}
\end{aligned}
$$

De $B_{G}$ Dy $B_{G^{*}}$ resulta $S_{1}=\left(\begin{array}{c}\left\{x_{1}, x_{2}, x_{3}, x_{4}, x_{5}, x_{6}, x_{7}, x_{8}, x_{9}, x_{10}\right\} \\ \left\{x_{1}, x_{2}, x_{3}, x_{4}, x 5, x_{6}, x_{7}, x_{8}, x_{9}, x_{10}\right\}\end{array}\right)$. Esta sustitución es estable. De ella se o0btienen $10 !=3628000$ sustituciones simples. Es difícil verificar el cumplimiento de la condición (1) para estas sustituciones. Las matrices $C$ y $D$ facilitan la solución del problema. Supongamos que $x_{1} \leftrightarrow x_{2}$ y construimos la tabla general $\mathrm{T}_{7}$ de las matrices $C$.

$$
\begin{aligned}
& \begin{array}{cccc}
G & x_{2} x_{3} x_{4} x_{5} x_{6} x_{7} x_{8} x_{9} x_{10} & G^{*} & x_{2} x_{3} x_{4} x_{5} x_{6} x_{7} x_{8} x_{9} x_{10} \\
x_{1} & 011001000 & x_{1} & 100000101
\end{array} \\
& T_{7} \\
& \Downarrow \\
& \tilde{S}_{2}=\left(\begin{array}{c}
x_{1}\left\{x_{2}, x_{5}, x_{6}, x_{8}, x_{9}, x_{10}\right\}\left\{x_{3}, x_{4}, x_{7}\right\} \\
x_{1}\left\{x_{3}, x_{4}, x_{5}, x_{6}, x_{7}, x_{9}\right\}\left\{x_{2}, x_{8}, x_{10}\right\}
\end{array}\right) \\
& \Downarrow \\
& \begin{array}{cccccc}
G & x_{2} x_{5} x_{6} x_{8} x_{9} x_{10} & x_{3} x_{4} x_{7} & G^{*} & x_{3} x_{4} x_{5} x_{6} x_{7} x_{9} & x_{2} x_{8} x_{10} \\
M_{1}=\left\{x_{2}, x_{5}, x_{6}, x_{8}, x_{9}, x_{10}\right\} & 222222 & 222 & M_{1}^{*}=\left\{x_{3}, x_{4}, x_{5}, x_{6}, x_{7}, x_{9}\right\} & 222222 & 222 \\
M_{2}=\left\{x_{3}, x_{4}, x_{7}\right\} & 221122 & 111 & M_{2}^{*}=\left\{x_{2}, x_{8}, x_{10}\right\} & 221122 & 111 \\
\sum_{\tau} & 443344 & 333 & & 443344 & 333
\end{array}
\end{aligned}
$$




$$
\begin{aligned}
& T_{8} \\
& \tilde{S}_{3}=\left(\begin{array}{l}
x_{1}\left\{x_{2}, x_{5}, x_{9}, x_{10}\right\}\left\{x_{6}, x_{8}\right\}\left\{x_{3}, x_{4}, x_{7}\right\} \\
x_{1}\left\{x_{3}, x_{4}, x_{7}, x_{9}\right\}\left\{x_{5}, x_{6}\right\}\left\{x_{2}, x_{8}, x_{10}\right\}
\end{array}\right) \\
& \Downarrow \\
& \begin{array}{cccccccc}
G & x_{2} x_{5} x_{9} x_{10} & x_{6} x_{8} & x_{3} x_{4} x_{7} & G^{*} & x_{3} x_{4} x_{7} x_{9} & x_{5} x_{6} & x_{2} x_{8} x_{10} \\
M_{1}=\left\{x_{2}, x_{5}, x_{9}, x_{10}\right\} & 2211 & 22 & 222 & M_{1}^{*}=\left\{x_{3}, x_{4}, x_{7}, x_{9}\right\} & 2211 & 22 & 222 \\
M_{2}=\left\{x_{6}, x_{8}\right\} & 2222 & 22 & 111 & M_{2}^{*}=\left\{x_{5}, x_{6}\right\} & 2222 & 22 & 111 \\
M_{3}=x_{3}, x_{4}, x_{7}\{\} & 2222 & 11 & 111 & M_{3}^{*}=\left\{x_{2}, x_{8}, x_{10}\right\} & 2222 & 11 & 111 \\
\sum_{\tau} & 6655 & 55 & 444 & & 6655 & 55 & 444
\end{array} \\
& T_{9} \\
& \Downarrow \\
& \widetilde{S}_{4}=\left\{\begin{array}{l}
x_{1}\left\{x_{2}, x_{5}\right\}\left\{x_{9}, x_{10}\right\}\left\{x_{6}, x_{8}\right\}\left\{x_{3}, x_{4}\right\} \\
x_{1}\left\{x_{3}, x_{4}\right\}\left\{x_{7}, x_{9}\right\}\left\{x_{5}, x_{6}\right\}\left\{x_{2}, x_{10}\right\}
\end{array}\right\}
\end{aligned}
$$$$
\Downarrow
$$$$
\begin{array}{cccccccccc}
G & x_{2} x_{5} & x_{9} x_{10} & x_{6} x_{8} x & x_{3} x_{4} x_{7} & G^{*} & x_{3} x_{4} & x_{7} x_{9} & x_{5} x_{6} & x_{2} x_{8} x_{10} \\
M_{1}=\left\{x_{2}, x_{5}\right\} & 22 & 11 & 22 & 221 & M_{1}^{*}=\left\{x_{3}, x_{4}\right\} & 22 & 11 & 22 & 212 \\
M_{2}=\left\{x_{9}, x_{10}\right\} & 11 & 11 & 22 & 221 & M_{2}^{*}=\left\{x_{7}, x_{9}\right\} & 11 & 11 & 22 & 212 \\
M_{3}=\left\{x_{6}, x_{8}\right\} & 22 & 22 & 22 & 111 & M_{3}^{*}=\left\{x_{5}, x_{6}\right\} & 22 & 22 & 22 & 111 \\
M_{4}=\left\{x_{3}, x_{4}, x_{7}\right\} & 22 & 22 & 11 & 111 & M_{4}^{*}=\left\{x_{2}, x_{8}, x_{10}\right\} & 22 & 22 & 11 & 111 \\
\sum_{\tau} & 77 & 66 & 77 & 664 & & 77 & 66 & 77 & 646
\end{array}
$$$$
T_{10}
$$$$
\Downarrow
$$$$
\tilde{S}_{5}=\left\{\begin{array}{l}
x_{1} x_{7}\left\{x_{2}, x_{5}\right\}\left\{x_{9}, x_{10}\right\}\left\{x_{6}, x_{8}\right\}\left\{x_{3}, x_{4}\right\} \\
x_{1} x_{8}\left\{x_{3}, x_{4}\right\}\left\{x_{7}, x_{9}\right\}\left\{x_{5}, x_{6}\right\}\left\{x_{2}, x_{10}\right\}
\end{array}\right\}
$$ 


$$
\begin{array}{cccccccccc}
\multicolumn{10}{c}{\Downarrow} \\
G & x_{2} x_{5} & x_{9} x_{10} & x_{6} x_{8} & x_{3} x_{4} & G^{*} & x_{3} x_{4} & x_{7} x_{9} & x_{5} x_{6} & x_{3} x_{10} \\
x_{1} & 00 & 00 & 00 & 11 & x_{1} & 00 & 00 & 00 & 11 \\
x_{7} & 00 & 11 & 00 & 00 & x_{8} & 00 & 11 & 00 & 00
\end{array}
$$

$T_{11}$

Las sustituciones $\widetilde{S}_{2}, \widetilde{S}_{3}, \widetilde{S}_{4}$ son inestables. De $T_{10}$ resulta que $x_{7}$ de $G$ y $x_{8}$ de $G^{*}$ son simples. Según $T_{10}$ se obtiene ,la susutitución $\widetilde{S}_{5}$. Construimos la tabla $T_{11}$ de las matrices $C$. De $T_{11}$ resulta que no hay otros vértices simplesy por eso, según $\widetilde{S}_{5}$ construimos la tabla $T_{12}$ de las matrices $D$. De $T_{12}$ se ve que $\widetilde{S}_{5}$ se queda sin cambio y por so es estable; máx $\sum_{\tau}=7$. Esto corresponde a $x_{2}, x_{5}, x_{6}, x_{8}$ de $G$ y $x_{3} x_{4} x_{5} x_{6}$ de $G^{*}$. Sea $x_{2} \leftrightarrow x_{3}$. Entonces de $\widetilde{S}_{5}$ resulta que $x_{5} \leftrightarrow x_{4}$ y se obtiene la sustitución $\widetilde{S}_{6}$. Según esta sustitución construimos la tabla $T_{13}$ de las matrices $D$.

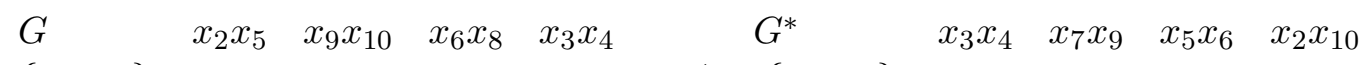

$$
\begin{aligned}
& M_{1}=\left\{x_{2}, x_{5}\right\} \quad 22 \quad 11 \quad 22 \quad 22 \quad M_{1}^{*}=\left\{x_{3}, x_{4}\right\} \quad 22 \quad 11 \quad 22 \quad 22 \\
& M_{2}=\left\{x_{9}, x_{10}\right\} \quad 11 \quad 11 \quad 22 \quad 22 \quad M_{2}^{*}=\left\{x_{7}, x_{9}\right\} \quad 11 \quad 11 \quad 22 \quad 22 \\
& M_{3}=\left\{x_{6}, x_{8}\right\} \quad 22 \quad 22 \quad 22 \quad 11 \quad M_{3}^{*}=\left\{x_{5}, x_{6}\right\} \quad 22 \quad 22 \quad 22 \quad 11 \\
& M_{4}=\left\{x_{3}, x_{4}\right\} \quad 22 \quad 22 \quad 11 \quad 11 \quad M_{4}^{*}=\left\{x_{2}, x_{10}\right\} \quad 22 \quad 22 \quad 11 \quad 11
\end{aligned}
$$

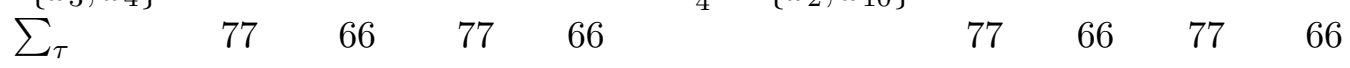

$$
\begin{aligned}
& T_{12} \\
& \tilde{S}_{6}=\left(\begin{array}{l}
x_{1} x_{7} x_{2} x_{5}\left\{x_{9}, x_{10}\right\}\left\{x_{6}, x_{8}\right\}\left\{x_{3}, x_{4}\right\} \\
x_{1} x_{8} x_{3} x_{4}\left\{x_{7}, x_{9}\right\}\left\{x_{5}, x_{6}\right\}\left\{x_{2}, x_{10}\right\}
\end{array}\right) \\
& \begin{array}{cccccccc}
G & x_{9} x_{10} & x_{6} x_{8} & x_{3} x_{4} & G^{*} & x_{7} x_{9} & x_{5} x_{6} & x_{2} x_{10} \\
x_{1} & 00 & 00 & 11 & x_{1} & 00 & 00 & 11 \\
x_{7} & 11 & 00 & 00 & x_{8} & 11 & 00 & 00 \\
x_{2} & 00 & 01 & 01 & x_{3} & 00 & 01 & 10 \\
x_{5} & 00 & 10 & 10 & x_{4} & 00 & 10 & 01 \\
x_{6} & 10 & & & x_{5} & 01 & & \\
x_{8} & 01 & & & x_{6} & 10 & & \\
x_{3} & 10 & & & x_{10} & 01 & & \\
x_{4} & 01 & & & x_{2} & 10 & & \\
x_{9} & & & & x_{9} & & & \\
x_{10} & & & & x_{7} & & &
\end{array}
\end{aligned}
$$


$T_{13}$

$\widetilde{S}_{6}$ es inestable. Las matrices $C$ engendran vértices simples $x_{6}, x_{8}, x_{3}, x_{4}$ en $G$ y $x_{5}, x_{6}, x_{10}, x_{12}$ en $G^{*}$. se obtiene la sustitución

$$
\widetilde{S}_{7}=\left(\begin{array}{c}
x_{1} x_{7} x_{2} x_{5} x_{6} x_{8} x_{3} x_{4}\left\{x_{9}, x_{10}\right\} \\
x_{1} x_{8} x_{3} x_{4} x_{5} x_{6} x_{10} x_{2}\left\{x_{7}, x_{9}\right\}
\end{array}\right)
$$

Según esta sutitución construimos la tabla general de las matrices $C$ (está incluida en $\left.T_{13}\right)$.

Obtenemos otros vértices simples: $x_{9}, x_{7}$ en $G$ y $x_{9}, x_{7}$ en $G^{*}$. De $T_{13}$ resulta

$$
S_{1}=\left(\begin{array}{c}
x_{1} x_{2} x_{3} x_{4} x_{5} x_{6} x_{7} x_{8} x_{9} x_{10} \\
x_{1} x_{3} x_{10} x_{2} x_{4} x_{5} x_{8} x_{6} x_{9} x_{7}
\end{array}\right)
$$

Renombrando los vértices de $G$ conforme a $S_{1}$ se obtiene $A_{G}\left(S_{1}\right)=A_{G^{*}}$ que significa que los grafos son isoformos.

En unos casos es necesario hallar todas las sustituciones para las cuales se cumple (1).

Supongamos que $\widetilde{S}$ es estable. Veamos unas propiedades de las sustitución (5).

Teorema 1

Sea $\{S\}$ el conjunto de todas las sutituciones $S$ según las cvuales $G \cong G^{*}$. Entonces el úmero de susutituciones de este conjunto que contiene la correspondencia $x_{i} \leftrightarrow x_{j}$ es igual al número de sustituciones del mismo conjunto que contienen la correspondencia $x_{i} \leftrightarrow x k$

para $\forall i, j, k \in(1, \ldots, n)$.

Demostración

Sin restringir la generalidad de la demostración podemos considerar que en $\widetilde{S} x_{b_{1}}=$ $x_{i}, x_{e_{1}}=x_{j}, x_{e_{2}}=x_{k}$. Todas las sustituciones de $\{S\}$ se obtienen de $\widetilde{S}$ por medio de las matrices $C$ y $D$. Designemos por $m_{1}$ el número de sustituciones que contiene la correspondencia $x_{i} \leftrightarrow x_{j}$ y por $m_{2}$ - las que contienen $x_{i} \leftrightarrow x_{k}$. Para hallar las sustituciones indicadas tenemos que considerar $x_{b_{1}}$ y $x_{e_{1}}$ simples en un caso y $x_{b_{1}}$ y $x_{e_{2}}$ en el otro. Respectivamente se obtienen las sustituciones

$$
\begin{aligned}
& \left(\begin{array}{c}
x_{a_{1}} \ldots x_{a_{t}} x_{b_{1}}\left\{x_{b_{2}}, x_{b_{3}}, \ldots, x_{b_{k}}\right\} \\
\ldots\left\{x_{c_{1}}, \ldots, x_{c_{s}}\right\} \\
x_{d_{1}} \ldots x_{d_{t}} x_{e_{1}}\left\{x_{e_{2}}, x_{e_{3}}, \ldots, x_{e_{k}}\right\} \\
\ldots\left\{x_{f_{1}}, \ldots, x_{f_{s}}\right\}
\end{array}\right) \\
& \left(\begin{array}{l}
x_{a_{1}} \ldots x_{a_{t}} x_{b_{1}}\left\{x_{b_{2}}, x_{b_{3}}, \ldots, x_{b_{k}}\right\} \\
x_{d_{1}} \ldots x_{d_{t}} x_{e_{2}}\left\{x_{e_{2}}, x_{e_{3}}, \ldots, x_{e_{k}}\right\} \ldots\left\{x_{c_{1}}, \ldots, x_{c_{s}}\right\} \\
\left.\ldots, x_{f_{1}}, \ldots, x_{f_{s}}\right\}
\end{array}\right)
\end{aligned}
$$

Por medio de las matrices $C$ y $D$ de (8) obtendremos las sustituciones $S_{1}^{(8)}, \ldots, S_{m_{1}}^{(8)}$ y de (9) a las otras $S_{1}^{(9)}, \ldots, S_{m_{2}}^{(9)}$. Compongamos la sustitución

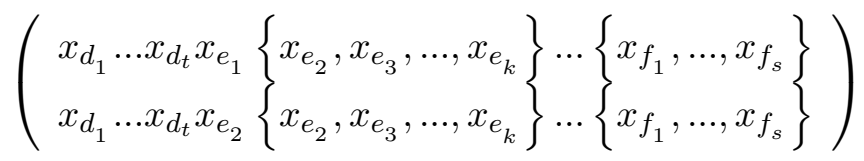


Por medio de las matrices $C$ y $D$ de (10) hallamos una sutitución simple $S_{T}$ que satisfaga la condición

$$
A_{G^{*}}=\left(S_{T}\right)=A_{G^{*}}
$$

y la llamaremos sustitución de transisción.

De (8),(9),(10) resulta que todas las sustituciones simples de (9), es decir las que contienen la correspondencia $x_{b_{1}} \leftrightarrow x_{e_{2}}$, se obtienen por medio de la multiplicación de las susutituciones simples de (8), es decir las que contienen la correspondencia $x_{b_{1}} \leftrightarrow x_{e_{1}}$, por la sustitución de transición $S_{T}$. Para esta susstitución existe la sustitución inversa $S_{T}^{-1}$. al multiplicar cada una de las sustituciones simples de (9) por esta sustitución obtendremos $m_{2}$ sustituciones que contienen la correspondencia $x_{b_{1}} \leftrightarrow x_{e_{1}}$.Pero tales sustituciones son $m_{1}$. Por consiguiente $m_{1}=m_{2}$. Lo que se trata de demostrar.

Si $S_{T}$ no existe entonces tampoco existe la correspondencia $x_{b_{1}} \leftrightarrow x_{e_{2}}$

De (10) en general pueden obtenerse varias sustituciones de transición.

Teorema 2

Cualesquiera dos sustituciones de transición engendran el mismo conjunto de sustituciones simples.

Demostración

Sea $S_{T}^{(1)}$ y $S_{T}^{(2)}$ dos diferentes sustituciones de transición. Supongamos que de (8) se obtienen $m_{1}$ sustituciones simples $S_{1}, \ldots, S_{m_{1}}$ que contienen la correspndencia $x_{b_{1}} \leftrightarrow x_{e_{1}}$. Multiplicamos cada una de estas sustituciones por las sustituciones de transición

$$
S_{1} \cdot S_{T}^{(1)}=S_{1}^{(1)}, \ldots, S_{m_{1}} \cdot S_{T}^{(1)}=S_{m_{1}}^{(1)}, S_{1} \cdot S_{T}^{(2)}=S_{1}^{(2)}, \ldots, S_{m_{1}} \cdot S_{T}^{(2)}=S_{m_{1}}^{(2)}
$$

Obtuvimos dos conjuntos de substituciones $\left\{S_{1}^{(1)}, \ldots, S_{m_{1}}^{(1)}\right\}$ y $\left\{S_{1}^{(2)}, \ldots, S_{m_{1}}^{(2)}\right\}$ que contienen la correspondencia $x_{b_{1}} \leftrightarrow x_{e_{2}}$. Componemos la unión de estos conjuntos y designamos por $m^{*}$ el número de elementos de esta unión. Multiplicamos las sustituciones del conjunto obtenido por la sustitución inversa de una de las sustituciones de transición. Obtendremos $m^{*}$ sustituciones que contengan la correspondencia $x_{b_{1}} \leftrightarrow x_{e_{1}}$. Según la condición existente $m_{1}$ de tales sustituciones Por consiguiente $m^{*}=m_{1}$, es decir $\left|\left\{S_{1}^{(1)}, \ldots, S_{m_{1}}^{(1)}\right\} U\left\{S_{1}^{(2)}, \ldots, S_{m_{1}}^{(2)}\right\}\right|=m_{1}$. La última igualdad puede cumplirse solamente cuando $\left\{S_{1}^{(1)}, \ldots, S_{m_{1}}^{(1)}\right\}=\left\{S_{1}^{(2)}, \ldots, S_{m_{1}}^{(2)}\right\}$, lo que se trataba de demostrar.

Designemos por $\left\{S_{x_{i} \leftrightarrow x_{j}}\right\}$ el conjunto de sustituciones simples que contienen la correspondencia $x_{i} \leftrightarrow x_{j}$ y que se obtienen de $\widetilde{S}$ por medio de las matrices $C$ y $D$ y con el cumplimiento de (1). En la base de los Teoremas 1 y 2 se puede demostrar

Teorema 3

Para cualesquiera dos vértices $x_{i} \in M_{r}, x_{j} \in M_{g}$ para $\forall r, g \in\{1, \ldots, q\}$ se verifica

$$
\forall x_{k} \in M_{r}^{*} U\left\{S_{x_{i} \leftrightarrow x_{k}}\right\}=\forall x_{h} \in M_{g}^{*} U\left\{S_{x_{j} \leftrightarrow x_{h}}\right\}
$$

De los Teoremas 1-3 resulta que el número $m$ de todas las sustituciones simples que se obtienen de $\widetilde{S}$ y para las cuales se cumple (1) satisface la condición

$$
m \leq\left|\left\{S_{x_{i} \leftrightarrow x_{k}}\right\}\right| \cdot\left|M_{j}\right|(13)
$$


para $\forall x_{i} \in M_{j}, \forall x_{k} \in M_{j}^{*}, j=1, \ldots, q$ para los cuales $\left\{S_{x_{i} \leftrightarrow x_{k}}\right\} \neq \emptyset$.

Según el Teorema 3 para investigar los grafos sobre isomorfismo es suficiente usar solamente un vértice de cualquier subconjunto $M_{j}$. Del Terorma 2 resulta que es suficiente buscar solamente una sustitución de transición. Vemos el caso más general. Supongamos que se conocen todas las sustituciones simples que contienen $x_{b_{1}} \leftrightarrow x_{e_{1}}, \ldots, x_{b_{1}} \leftrightarrow x_{e_{r}}$ y es necesario hallar todas las sustituciones que contienen $x_{b_{1}} \leftrightarrow x_{e_{r+1}}, \ldots, x_{b_{1}} \leftrightarrow x_{e_{2 r}}$. Componemos la sustitución múltiple

$$
\left(\begin{array}{c}
x_{d_{1}} \ldots x_{d_{t}}\left\{x_{e_{1}}, \ldots, x_{e_{r}}\right\}\left\{x_{e_{r+1}}, \ldots, x_{e_{2 r}}, \ldots, x_{e_{k}}\right\} \ldots\left\{x_{f_{1}} \ldots x_{f_{s}}\right\} \\
x_{d_{1}} \ldots x_{d_{t}}\left\{x_{e_{r+1}}, \ldots, x_{e_{2 r}}\right\}\left\{x_{e_{1}}, \ldots, x_{e_{r}}, x_{e_{2 r+1}}, \ldots, x_{e_{k}}\right\} \ldots\left\{x_{f_{1}} \ldots x_{f_{s}}\right\}
\end{array}\right)
$$

Cualquier sustitución simple $S_{T}$ que se obtiene de (14) por medio de las matrices $C$ y $D$ y que satisface la condición (11) será una sustitukción de transición de las sustituciones que contienen las correspondencias $x_{b_{1}} \leftrightarrow x_{e_{1}}, \ldots, x_{b_{1}} \leftrightarrow x_{e_{r}}$ a las sustituciones que contienen las correspondencias $x_{b_{1}} \leftrightarrow x_{e_{r+1}}, \ldots, x_{b_{1}} \leftrightarrow x_{e_{2 r}}$. Estas últimas se obtienen multiplicando las sustituciones conocidas por $S_{T}$.

Veamos una de las aplicaciones del método expuesto.

Supongamos que en el grafo $G$ se conoce un ciclo hamiltoniano $x_{i_{1}}, \ldots, x_{i_{n}}, x_{i_{1}}$

Componemos la sustitución $S=\left(\begin{array}{c}x_{i_{1}} \ldots x_{i_{n}} \\ x_{1} \ldots x_{n}\end{array}\right)$ Renombramos los vértices del grafo $G$ según $S$. Obtendremos un grafo $G^{*}$ tal que $G \cong G^{*}$. La sucesión $x_{i_{1}}, \ldots, x_{i_{n}}, x_{i_{1}}$ forma un ciclo hamiltoniano en $G^{*}$. Hallemos todas las sustituciones simples para las cuales se cumple (1). Arreglando los vértices de la segunda fila en caada una de éstas sustituciones de acuerdo con el ciclo $x_{1}, \ldots, x_{n}, x_{1}$. Obtendremos en las primeras filas ciclos hamiltonianos en G.De ésta manera obtendremos otros ciclos.

Hace falta señalar que dos substituciones diferentes pueden determinar el mismo ciclo. Así, por ejemplo, las sustituciones $\left(\begin{array}{c}x_{1} x_{4} x_{2} x_{5} x_{6} x_{8} x_{10} x_{7} x_{9} x_{3} \\ x_{1} x_{2} x_{3} x_{4} x_{5} x_{6} x_{7} x_{8} x_{9} x_{10}\end{array}\right)$ y $\left(\begin{array}{c}x_{8} x_{10} x_{7} x_{9} x_{3} x_{1} x_{4} x_{2} x_{5} x_{6} \\ x_{1} x_{2} x_{3} x_{4} x_{5} x_{6} x_{7} x_{8} x_{9} x_{10}\end{array}\right)$ para las cuales se cumplen las condiciones (1) para los grafos del ejemplo 2 que determinan en el $G$ el mismo ciclo $x_{1} x_{4} x_{2} x_{5} x_{6} x_{8} x_{10} x_{7} x_{9} x_{3}$.

Todavía no se conocen las condiciones cuando el conjunto $\{S\}$ determina todos los ciclos hamiltonianos. Lo expuesto sobre ciclos es cierto y para cadenas hamiltonianas.

Veamos el problema del destacado matemático alemán G, Frobenius.

Dadas dos matrices $A=\left(a_{i j}\right)_{n \times n}$ y $A^{*}=\left(a_{i j}^{*}\right)_{n \times n}$ con elementos arbitrarios. Las matrices se llaman fuertemente equivalentes si existe una sustitución de filas y columnas que transforme una matriz a otra. El problema consiste en la búsqueda de esta sustitución. Es fácil mostrar condiciones necesarias evidentes de la equivalencia de dos matrices: ambas matrices deben contener los mismos elementos, las diagonales principales deben contener los mismos elementos y en cantidades iguales, para cada fila (columna) de una matriz existe una fila (columna) en otra matriz con los mismos elementos y en cantidades iguales, etc. Con tales condiciones en la mayoría de los casos el problema no se resuelve. Vamos a reducir éste problema al problema del isimorfismo de dos grafos. Las matrices $A$ y $A^{*}$ se consideran como matrices de los pesos de los arcos de los grafos completos de Berge. Dos matrices son fuertemente equivalentes si y sólo si los grafos pesados respectivos de Berge son isomorfos en el sentido siguiente: si $x_{i} \leftrightarrow x_{j}$ y $x_{k} \leftrightarrow x_{s}$ entonces $a_{i k}=a_{i s}^{*}$ [8]. Para 
investigar los grafos sobre isomorfismo podemos usar las matrices $C$ y $D$.

Ejemplo 3. Investigar si las matrices $A$ y $A^{*}$ son fuertemente equivalentes.

$$
\begin{aligned}
& \begin{array}{llllllllll}
x_{1} & x_{2} & x_{3} & x_{4} & x_{5} & x_{6} & x_{7} & x_{8} & x_{9} & x_{10}
\end{array} \\
& x_{1} \quad\left[\begin{array}{llllllllll}
\alpha & \alpha & \beta & \beta & \gamma & \gamma & \alpha & \beta & \gamma & \alpha
\end{array}\right] \\
& \begin{array}{l|llllllllll}
x_{2} & \alpha & \beta & \alpha & \gamma & \beta & \gamma & \alpha & \alpha & \beta & \gamma
\end{array} \\
& \begin{array}{l|llllllllll}
x_{3} & \beta & \alpha & \beta & \gamma & \gamma & \alpha & \beta & \gamma & \alpha & \alpha
\end{array} \\
& \begin{array}{l|llllllllll}
x_{4} & \gamma & \beta & \gamma & \alpha & \beta & \alpha & \alpha & \gamma & \beta & \alpha
\end{array} \\
& A=\begin{array}{l|llllllllll}
x_{5} & \beta & \gamma & \gamma & \alpha & \alpha & \beta & \alpha & \beta & \alpha & \gamma \\
x_{6} & \gamma & \gamma & \alpha & \beta & \alpha & \beta & \beta & \alpha & \gamma & \alpha
\end{array} \\
& \begin{array}{l|llllllllll}
x_{7} & \alpha & \alpha & \beta & \alpha & \alpha & \beta & \gamma & \gamma & \gamma & \beta
\end{array} \\
& \begin{array}{l|llllllllll}
x_{8} & \beta & \alpha & \gamma & \gamma & \beta & \alpha & \gamma & \alpha & \alpha & \beta
\end{array} \\
& \begin{array}{l}
x_{9} \\
x_{10}
\end{array}\left[\begin{array}{llllllllll}
\gamma & \beta & \alpha & \beta & \alpha & \gamma & \gamma & \alpha & \alpha & \beta \\
\alpha & \gamma & \alpha & \alpha & \gamma & \alpha & \beta & \beta & \beta & \gamma
\end{array}\right] \\
& \begin{array}{llllllllll}
x_{1} & x_{2} & x_{3} & x_{4} & x_{5} & x_{6} & x_{7} & x_{8} & x_{9} & x_{10}
\end{array}
\end{aligned}
$$

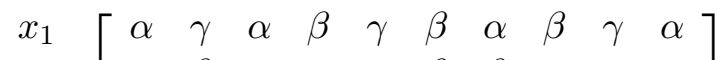

$$
\begin{aligned}
& \begin{array}{l|llllllllll}
x_{2} & \gamma & \beta & \gamma & \alpha & \alpha & \beta & \beta & \gamma & \alpha & \alpha
\end{array} \\
& \begin{array}{l|llllllllll}
x_{3} & \beta & \gamma & \alpha & \alpha & \beta & \gamma & \alpha & \gamma & \alpha & \beta
\end{array} \\
& \begin{array}{l|llllllllll}
x_{4} & \alpha & \alpha & \beta & \beta & \gamma & \gamma & \beta & \alpha & \alpha & \gamma
\end{array} \\
& A^{*}=\begin{array}{l|llllllllll}
x_{5} & \beta & \alpha & \gamma & \gamma & \beta & \alpha & \alpha & \alpha & \gamma & \beta \\
x_{6} & \gamma & \beta & \beta & \gamma & \alpha & \alpha & \alpha & \beta & \alpha & \gamma
\end{array} \\
& \begin{array}{l|llllllllll}
x_{7} & \alpha & \beta & \alpha & \beta & \alpha & \alpha & \gamma & \gamma & \beta & \gamma
\end{array} \\
& \begin{array}{l|llllllllll}
x_{8} & \beta & \gamma & \gamma & \alpha & \alpha & \beta & \gamma & \alpha & \beta & \alpha \\
x_{9} & \gamma & \alpha & \alpha & \alpha & \gamma & \alpha & \beta & \beta & \gamma & \beta
\end{array} \\
& x_{10}\left[\begin{array}{llllllllll}
\alpha & \alpha & \beta & \gamma & \beta & \gamma & \gamma & \alpha & \beta & \alpha
\end{array}\right]
\end{aligned}
$$

Solución. Para las diagonales principales de las matrices se cumplen las condiciones necesarias. Todas las filas y columnas de ambas matrices están compuestas de los mismos elementos en las mismas cantidades. Por eso la primera sustitución múltiple es

$$
\widetilde{S}_{1}=\left(\begin{array}{l}
\left\{x_{1}, x_{2}, x_{3}, x_{4}, x_{5}, x_{6}, x_{7}, x_{8}, x_{9}, x_{10}\right\} \\
\left\{x_{1}, x_{2}, x_{3}, x_{4}, x_{5}, x_{6}, x_{7}, x_{8}, x_{9}, x_{10}\right\}
\end{array}\right)
$$

Sea $X_{1} \leftrightarrow X_{1}$ Construimos la tabla general $\mathrm{T}_{14}$ de las matrices $C$.

$$
\begin{aligned}
& A \quad x_{2} x_{3} x_{4} x_{5} x_{6} x_{7} x_{8} x_{9} x_{10} \quad A^{*} \quad x_{2} x_{3} x_{4} x_{5} x_{6} x_{7} x_{8} x_{9} x_{10} \\
& \begin{array}{llll}
x_{1} & \alpha \beta \beta \gamma \gamma \alpha \beta \gamma \alpha & x_{1} & \gamma \alpha \beta \gamma \beta \alpha \beta \gamma \alpha
\end{array} \\
& T_{14} \\
& \tilde{S}=\left(\begin{array}{c}
x_{1}\left\{x_{2}, x_{7}, x_{10}\right\}\left\{x_{3}, x_{4}, x_{8}\right\}\left\{x_{5}, x_{6}, x_{9}\right\} \\
x_{1}\left\{x_{3}, x_{7}, x_{10}\right\}\left\{x_{4}, x_{6}, x_{8}\right\}\left\{x_{2}, x_{5}, x_{9}\right\}
\end{array}\right)
\end{aligned}
$$

Construimos la tabla general $\mathrm{T}_{15}$ de las matrices $D$. 


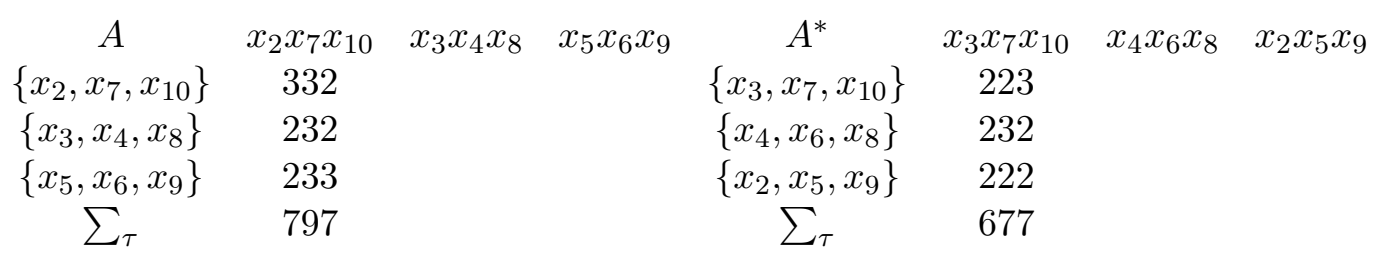

$T_{15}$

Comparando el primer par de matrices observamos que las condiciones (4) no se cumplen. Por consiguiente $x_{1}$ no puede corresponder a $x_{1}$ y no es necesario calcular los elementos de otras matrices. Sea $x_{1} \leftrightarrow x_{2}$. Construimos la tabla $\mathrm{T}_{16}$ de las matrices $C$.

$$
\begin{array}{cccc}
A & x_{2} x_{3} x_{4} x_{5} x_{6} x_{7} x_{8} x_{9} x_{10} & A^{*} & x_{1} x_{3} x_{4} x_{5} x_{6} x_{7} x_{8} x_{9} x_{10} \\
x_{1} & \alpha \beta \beta \gamma \gamma \alpha \beta \gamma \alpha & x_{2} & \gamma \gamma \alpha \alpha \beta \beta \gamma \alpha \alpha
\end{array}
$$

\section{$T_{16}$}

Las condiciones (4) no se cumplen. Por eso $x_{1}$ no puede corresponder a $x_{2}$. Análogamente obtendremos que $x_{1}$ no corresponde a $x_{3}, x_{4}, x_{5}$. Sea $x_{1} \leftrightarrow x_{2}$. Sea $x_{1} \leftrightarrow x_{6}$. Construimos la tabla $T_{17}$.

$$
\begin{array}{cccc}
A & x_{2} x_{3} x_{4} x_{5} x_{6} x_{7} x_{8} x_{9} x_{10} & A^{*} & x_{2} x_{3} x_{4} x_{5} x_{6} x_{7} x_{8} x_{9} x_{10} \\
x_{1} & \alpha \beta \beta \gamma \gamma \alpha \beta \gamma \alpha & x_{2} & \gamma \beta \beta \gamma \alpha \alpha \beta \alpha \gamma
\end{array}
$$

$$
T_{17}
$$

Las condiciones (4) se cumplen. De $T_{17}$ resulta la sustitución múltple $\widetilde{S}_{3}$

$$
\tilde{S}_{3}=\left(\begin{array}{c}
X_{1}\left\{X_{2}, X_{7}, X_{10}\right\}\left\{X_{3}, X_{4}, X_{8}\right\}\left\{X_{5}, X_{6}, X_{9}\right\} \\
X_{6}\left\{X_{5}, X_{7}, X_{9}\right\}\left\{X_{2}, X_{3}, X_{8}\right\}\left\{X_{1}, X_{4}, X_{10}\right\}
\end{array}\right)
$$

Según esta sustitución construimos la tabla $T_{18}$ de las matrices $D$.

$\begin{array}{cccccccc}A & x_{2} x_{7} x_{10} & x_{3} x_{4} x_{8} & x_{5} x_{6} x_{9} & A^{*} & x_{5} x_{7} & x_{2} x_{3} x_{8} & x_{1} x_{4} x_{10} \\ \left\{x_{2}, x_{7}, x_{10}\right\} & 332 & 223 & 332 & \left\{x_{5}, x_{7}, x_{9}\right\} & 332 & 223 & 332 \\ \left\{x_{3}, x_{4}, x_{8}\right\} & 232 & 222 & 212 & \left\{x_{2}, x_{3}, x_{8}\right\} & 232 & 222 & 212 \\ \left\{x_{5}, x_{6}, x_{9}\right\} & 233 & 222 & 122 & \left\{x_{1}, x_{4}, x_{10}\right\} & 233 & 222 & 122 \\ \sum_{\tau} & 797 & 667 & 666 & \sum_{\tau} & 797 & 667 & 666\end{array}$


Las condiciones (4) se cumplen para cada par de matrices. De $T_{18}$ se obtiene

$$
\widetilde{S}_{4}=\left(\begin{array}{l}
x_{1} x_{2} x_{7} x_{10} x_{8} x_{5} x_{6} x_{9}\left\{x_{3}, x_{4}\right\} \\
x_{6} x_{5} x_{7} x_{9} x_{8} x_{1} x_{4} x_{10}\left\{x_{2}, x_{3}\right\}
\end{array}\right)
$$

Según la sustitución obtenida construimos la tabla $T_{19}$ de las matrices $C$

$\begin{array}{cccc}A & x_{3} x_{4} & A^{*} & x_{2} x_{3} \\ x_{1} & \beta \beta & x_{6} & \beta \beta \\ x_{2} & \alpha \gamma & x_{5} & \alpha \gamma \\ x_{7} & \beta \alpha & x_{7} & \beta \alpha \\ x_{10} & \alpha \alpha & x_{9} & \alpha \alpha \\ x_{8} & \gamma \gamma & x_{8} & \gamma \gamma \\ x_{5} & \gamma \alpha & x_{1} & \gamma \alpha \\ x_{6} & \alpha \beta & x_{4} & \alpha \beta \\ x_{9} & \alpha \beta & x_{10} & \alpha \beta \\ x_{3} & & x_{2} & \\ x_{4} & & x_{3} & \end{array}$

De $T_{19}$ resulta $S=\left(\begin{array}{l}x_{1} x_{2} x_{7} x_{10} x_{8} x_{5} x_{6} x_{9} x_{3} x_{4} \\ x_{6} x_{5} x_{7} x_{9} x_{8} x_{1} x_{4} x_{10} x_{2} x_{3}\end{array}\right)$. Para esta sustitución se cumple $A(S)=A^{*}$ que significa que las matrices son fuertemente equivalentes.

3. Funciones lógicas isomorfas

Dadas dos funciones lógicas $F_{1}\left(x_{1}, \ldots, x_{n}\right)$ y $F_{2}\left(x_{1}, \ldots, x_{n}\right)$. Si existe la sustitución $S_{x}=\left(\begin{array}{c}x_{i} \ldots x_{n} \\ x_{i j} \ldots x_{i_{n}}\end{array}\right)$ tal que por medio de ella una de las fnciones se transforma a otra, por ejemplo $F_{1}\left(x_{1}, \ldots, x_{n}\right)=F_{2}\left(x_{1}, \ldots, x_{n}\right)$, entonces se dice que estas funciones son isomorfas y se escribe $F_{1} \cong F_{2}$.Supongamos que las funciones se dan por los subconjuntos $M_{F_{1}}^{\varepsilon}=(i=1,2 ; \varepsilon \in\{0,1\})$ de juegos binarios de las variables $x_{i}, \ldots, x_{n} . M_{F_{1}}^{\varepsilon}=$ $\left\{\alpha: F_{1}(\alpha)=\varepsilon\right\}, M_{F_{2}}^{\varepsilon}=\left\{\beta: F_{2}(\beta)=\varepsilon\right\}, M_{F !}={ }_{F_{1}}^{1} M_{F_{1}}^{1} U M_{F_{1}}^{0}, M_{F_{2}}=M_{F_{2}}^{1} U M_{F_{2}}^{0}[1],[2]$

Veamos primero el caso cuando las funciones están definidas por complento. En este caso $\left|M_{F_{1}}\right|=\left|M_{F_{2}}\right|=2^{n}$ y por eso es suficiente examinarr solamente $M_{F_{1}}^{1}$ y $M_{F_{2}}^{1}$. Designemos por $y_{1}, \ldots, y_{m}$ los juegos binarios de los subconjuntos indicados y componemos las matrices $A_{F_{1}}=\left(a_{i j}\right)_{n \times n}, A_{F_{2}}=\left(a_{i j}^{*}\right)$, donde $a_{i j}\left(a_{i j}^{*}\right)$ es igual al valor de $x_{j}$ en el juego $y_{i}$ de $F_{1}\left(F_{2}\right)$.

$$
\begin{array}{ccccccc}
x_{1} & \cdot & \cdot & \cdot & x_{n} & & \\
& y_{1} & a_{11} & \cdot & \cdot & & \\
& \cdot & \cdot & \cdot & a_{1 n} \\
A_{F !} & \cdot & \cdot & \cdot & \cdot & \cdot & \cdot \\
& \cdot & \cdot & \cdot & \cdot & \cdot & \cdot \\
& y_{m} & a_{m 1} & \cdot & \cdot & \cdot & \cdot \\
x_{1} & \cdot & \cdot & \cdot & x_{n} & & \\
\end{array}
$$




$$
\begin{array}{rcccccc} 
& y_{1} & a_{11}^{*} & \cdot & \cdot & \cdot & a_{1 n}^{*} \\
& \cdot & \cdot & \cdot & \cdot & \cdot & \cdot \\
A_{F_{2}} & \cdot & \cdot & \cdot & \cdot & \cdot & \cdot \\
& \cdot & \cdot & \cdot & \cdot & \cdot & \cdot \\
& y_{m} & a_{m 1}^{*} & \cdot & \cdot & \cdot & \cdot \\
& & & \cdot
\end{array}
$$

Si las funciones son isomorfas entonces por medio de la sustitución $S_{x}$ el subconjunto $M_{F_{1}}^{1}$ se transforma al subconjunto $M_{F_{2}}^{1}$. En este escribiremos $M_{F_{1}}^{1}\left(S_{x}\right)=M_{F_{2}}^{1}$. El cumplimiento de la condición $A_{F_{1}}\left(S_{x}\right)=A_{F_{2}}$ no es obligatoria. Lo principal es que cada juego de $M_{F_{1}}^{1}$ se transforme a un juego de $M_{F_{2}}^{1}$ por medio de la sustitución indicada.

Por las matrices $A_{F_{1}}$ y $A_{F_{2}}$ construimos las matrices $B$ tanto por las columnas como y por las filas y las designaremos por $B_{F_{i}, x}$ y $B_{F_{i}, y}$ respectivamente. Según estas matrices hallamos las sustituciones $\widetilde{S}_{x}$ y $\widetilde{S}_{y}$ y después las matrices $C_{M_{i}, x}, C_{M_{i}, y}, D_{M_{1}, x}, D_{M_{1}, y}$. En las tablas generales de las matrices $C$ y $D$ se escriben tanto los subconjuntos de $\widetilde{S}_{x}$ como y los de $\widetilde{S}_{y}$.

Para hallar las sustituciones múltiples $\widetilde{S}_{x}$ y $\widetilde{S}_{y}$ podemos usar la descomposición de las funciones lógicas [2] tomando en calidad de funciones auxiliares $\Psi_{1}=x_{i}$ e $\Psi_{2}=x_{j}$ cuando se verifica la exiustencia de la correspondencia $x_{i} \leftrightarrow x_{j}$. A veces es útil usar el peso de la derivada [3], [4] como invariante.

Ejemplo 4 Investigar sobre isomorfismo las funciones $F_{1}\left(x_{1}, \ldots, x_{7}\right)$ y $F_{2}\left(x_{1}, \ldots, x_{7}\right)$ si $M_{F_{1}}^{1}=\{1011110,0001110,1110000,1010100,0010101,1110101,0101010,1001001\} M_{F_{2}}^{1}=$ $\{0101001,0001011,1000101,0011111,1001010,1101011,0010110,0110100\}$ Solución. Construimos las matrices $A_{F_{1}}$ y $A_{F_{2}}$.

$$
\begin{aligned}
& \begin{array}{lllllll}
x_{1} & x_{2} & x_{3} & x_{4} & x_{5} & x_{6} & x_{7}
\end{array} \\
& \begin{array}{l}
y_{1} \\
y_{2}
\end{array}\left[\begin{array}{lllllll}
1 & 0 & 1 & 1 & 1 & 1 & 0 \\
0 & 0 & 0 & 1 & 1 & 1 & 0 \\
1 & 1 & 1 & 0 & 0 & 0 & 0
\end{array}\right] \\
& \begin{array}{llllllll}
y_{3} & 1 & 1 & 1 & 0 & 0 & 0 & 0
\end{array} \\
& A_{F_{1}}=\begin{array}{l}
y_{4} \\
y_{5} \\
y_{6} \\
y_{7} \\
y_{8}
\end{array}\left[\begin{array}{lllllll}
1 & 0 & 1 & 0 & 1 & 0 & 0 \\
0 & 0 & 1 & 0 & 1 & 0 & 1 \\
1 & 1 & 1 & 0 & 1 & 0 & 1 \\
0 & 1 & 0 & 1 & 0 & 1 & 0 \\
1 & 0 & 0 & 1 & 0 & 0 & 1
\end{array}\right] \\
& \begin{array}{lllllll}
x_{1} & x_{2} & x_{3} & x_{4} & x_{5} & x_{6} & x_{7}
\end{array} \\
& A_{F_{2}}=\begin{array}{l}
y_{1} \\
y_{2} \\
y_{3} \\
y_{4} \\
y_{5} \\
y_{6} \\
y_{7} \\
y_{8}
\end{array}\left[\begin{array}{lllllll}
0 & 0 & 0 & 1 & 0 & 1 & 1 \\
0 & 0 & 1 & 1 & 1 & 1 & 1 \\
0 & 1 & 0 & 1 & 0 & 0 & 1 \\
1 & 0 & 0 & 0 & 1 & 0 & 1 \\
1 & 0 & 0 & 1 & 0 & 1 & 0 \\
1 & 1 & 0 & 1 & 0 & 1 & 1 \\
0 & 0 & 1 & 0 & 1 & 1 & 0 \\
0 & 1 & 1 & 0 & 1 & 0 & 0
\end{array}\right]
\end{aligned}
$$

Componemos grupos de filas y hallamos las matrices $B_{F_{1}, x}$ y $B_{F_{2}, y}$ 


$\begin{array}{ccccccc}x 1 & x 2 & x 3 & x 4 & x 5 & x 6 & x 7 \\ 0 & 0 & 0 & 1 & 1 & 1 & 0 \\ 1 & 1 & 1 & 0 & 0 & 0 & 0 \\ 1 & 0 & 1 & 0 & 1 & 0 & 0 \\ 0 & 0 & 1 & 0 & 1 & 0 & 1 \\ 0 & 1 & 0 & 1 & 0 & 1 & 0 \\ 1 & 0 & 0 & 1 & 0 & 0 & 1 \\ 1 & 0 & 1 & 1 & 1 & 1 & 0 \\ 1 & 1 & 1 & 0 & 1 & 0 & 1 \\ x_{1} & x_{2} & x_{3} & x_{4} & x_{5} & x_{6} & x_{7} \\ 0 & 0 & 1 & 1 & 0 & 1 & 1 \\ 0 & 1 & 1 & 1 & 0 & 0 & 1 \\ 1 & 0 & 0 & 0 & 1 & 0 & 1 \\ 1 & 0 & 1 & 1 & 0 & 1 & 0 \\ 0 & 0 & 0 & 0 & 1 & 1 & 0 \\ 0 & 1 & 0 & 0 & 1 & 0 & 0 \\ 0 & 0 & 1 & 1 & 1 & 1 & 1 \\ 1 & 1 & 1 & 0 & 0 & 1 & 1 \\ x_{1} & x_{2} & x_{3} & x_{4} & x_{5} & x_{6} & x_{7} \\ B_{F_{1}, x} & {\left[\begin{array}{cccccccc}3 & 2 & 3 & 3 & 3 & 2 & 2 \\ 2 & 1 & 2 & 1 & 1 & 1 & 1\end{array}\right]} \\ x_{1} & x_{2} & x_{3} & x_{4} & x_{5} & x_{6} & x_{7} \\ B_{F_{1}, y} & {\left[\begin{array}{lllllll}2 & 2 & 2 & 3 & 3 & 3 & 3 \\ 1 & 1 & 1 & 2 & 1 & 2 & 2\end{array}\right]}\end{array}$

De estas matrices resulta $\widetilde{S}_{x}=\left(\begin{array}{l}x_{4}\left\{x_{1}, x_{3}, x_{5}\right\}\left\{x_{2}, x_{6}, x_{7}\right\} \\ x_{5}\left\{x_{4}, x_{6}, x_{7}\right\}\left\{x_{1}, x_{2}, x_{3}\right\}\end{array}\right)$

Análogamente hallamos $B_{F_{1}, x}$ y $B_{F_{2}, y}, \widetilde{S}_{y}$. Construimos la tabla $\mathrm{T}_{20}$ de la matrices $C$. 